\title{
Proteomics for Clinical Assessment of Kidney Disease
}

\author{
Alberto Ortiz
}

Kidney disease is one of the fastest growing causes of death worldwide, disclosing an unmet clinical need for early diagnosis and optimized risk stratification that allows high risk patient selection for clinical trials and for more intensive nephroprotective interventions in the clinic. The current issue of PROTEOMICS - Clinical Applications contains four manuscripts that explore different aspects of clinical proteomics implementation in the context of acute kidney injury, chronic kidney disease and, more specifically, diabetic kidney disease, and kidney transplantation from a diagnostic and risk stratification point of view. Overall, the evidence discussed suggests that chronic kidney disease is an example where clinical proteomics has become a valuable tool ready for clinical implementation, expected to have a major impact in patient management.

Kidney disease is one of the fastest growing causes of death worldwide. Based on the Global Burden of Disease (GBD) study, it was recently estimated that at the current rate of growth, chronic kidney disease (CKD) would become the second cause of death in Spain in the next decades, and Spanish data are not very different from those worldwide. ${ }^{[1,2]}$ These estimates referred only to CKD, defined as evidence of kidney injury (e.g., pathological albuminuria or others) or decreased glomerular filtration rate (below $60 \mathrm{~mL} \mathrm{~min}^{-1}$ per $1.73 \mathrm{~m}^{2}$ ) persisting for more than 3 months, with implications for health. ${ }^{[3]}$ However, mortality due to acute kidney injury (AKI) was not assessed by GBD. Currently, there is no therapy for AKI beyond replacing renal function if required and where it is available. ${ }^{[4,5]}$ In this regard, CKD increases the risk of cardiovascular death as well as of AKI. Moreover, renal replacement therapy does not restore mortality risk in CKD patients back to baseline. ${ }^{[6]}$ This is due to insufficient replacement of just some renal functions in the case of dialysis (e.g., production of the antiaging factor Klotho, which is lost early in the course of CKD, is not replaced). ${ }^{[7]}$ Kidney transplantation is a better option, but still presents some issues such as clinical or subclinical kidney injury due to rejection or nephrotoxicity. In this regard, major advances

\section{Dr. A. Ortiz}

IIS-Fundacion Jimenez Diaz

School of Medicine

Universidad Autonoma de Madrid

Fundacion Renal Iñigo Alvarez de Toledo-IRSIN and REDINREN

Madrid 28040, Spain

E-mail: aortiz@fjd.es

See accompanying article by Julie Klein and Joost P Schanstra, https:// doi.org/10.1002/prca.201800089, David Marx et al., https://doi.org/10.10 02/prca.201800091, Justyna Siwy et al., https://doi.org/10.1002/prca.2018 00144, and Frederik Persson and Peter Rossing, https://doi.org/10. 1002/prca.201800176

The ORCID identification number(s) for the author(s) of this article can be found under https://doi.org/10.1002/prca.201900004

DOI: 10.1002/prca.201900004 are required in the way we diagnose, risk-stratify, and treat kidney disease to change the current trend toward increasing mortality from kidney disease. Systems biology offers the promise of both improved risk stratification and diagnostic tools that allow a precision medicine approach, as well as the possibility to identify novel pathogenic pathways and therapeutic targets. Of the diverse systems biology techniques, proteomics are closest to routine clinical application for kidney diseases. ${ }^{[8,9]}$ As an example, laser dissection mass spectrometry is currently the best available option to characterize the nature of glomerular amyloidosis, a requirement

for etiology-targeted therapy. ${ }^{[10]}$ In this issue of PROTEOMICSClinical Applications, four manuscripts explore different aspects of clinical proteomics in the context of AKI, CKD, and kidney transplantation from a diagnostic and risk stratification point of view. ${ }^{[11-14]}$ In this regard, another major application of proteomics to kidney research, the identification of novel pathogenic pathways, is not specifically addressed. ${ }^{[15]}$

Klein and Schanstra explore the significance of urine or kidney proteomics biomarkers identified in animal models to the clinical situation. ${ }^{[11]}$ An exhaustive literature review identified 68 relevant publications. However, they found little evidence that proteomic biomarkers identified from preclinical studies had actual clinical translation. In this regard, the availability of human urine allows the direct noninvasive characterization of proteomic biomarkers in human samples. The main interest of preclinical proteomic studies lay in the evaluation of drug efficacy and insights into drug mechanisms of action. Thus, an ingenuity pathway analysis using preclinical proteomics data from the literature identified acute phase response signaling as the top canonical pathway in AKI and cytoskeleton signaling in CKD, in accordance with current concepts about their pathogenesis in humans. Of special interest is the development of "humanized proteomic readouts" for their use in drug assessment. These readouts combine proteomic biomarkers shared by human and preclinical disease to allow the evaluation of drugs of potential clinical interest in preclinical models.

Persson and Rossing explore urinary proteomics in human CKD. ${ }^{[12]}$ Specifically, they focus on urinary proteomics using capillary electrophoresis coupled to mass spectrometry (CE-MS), a technique used to develop the CKD273 urine proteomics classifier. CKD273 has received a letter of support for CKD273 from the US Food and Drug Administration. ${ }^{[9]}$ Persson and Rossing review the available evidence regarding the potential clinical use of CKD273 as a diagnostic and risk stratification tool, including the ongoing clinical trial Proteomic prediction and Renin angiotensin aldosterone system Inhibition prevention 
Of early diabetic nephRopathy in TYpe 2 diabetic participants with normoalbuminuria (PRIORITY). Diabetic kidney disease continues to be the main cause of CKD-associated mortality and improved risk stratification tools are required to identify patients at highest risk of progression early in the course of the disease. ${ }^{[2]}$ PRIORITY has recruited 1777 participants, and $12.3 \%$ of these have a high-risk urine CKD273 proteomics pattern. ${ }^{[16]}$ These high-risk patients were randomized to spironolactone or placebo to test the hypothesis that urinary proteomics identification of high-risk patients may allow an intervention that prevents or delays the progression to microalbuminuria. More recently, it was shown that CKD273 may be optimized for the prediction of rapid progression when glomerular filtration rate in patients without pathological albuminuria, that is, in those individuals that similar to CKD patients, have a high risk of CKD progression, but that do not fulfill the current criteria to be diagnosed of CKD. ${ }^{[17]}$ This opens the door to future adjustments of the CKD definition through the use of urinary proteomics criteria.

In an original study, Siwy et al. explore the value of CKD273 and urinary proteomics in a randomized clinical trial of an antidiabetic agent, the dipeptidyl peptidase- 4 inhibitor linagliptin, in diabetic kidney disease (MARLINA-T2D)(13). CKD273 independently predicted CKD progression (i.e., annual eGFR decrease) and allowed the stratification of patients into high-risk and lowrisk of progression. Among high-risk patients, linagliptin prevented a significant loss of the renal function that was observed in placebo-treated high-risk patients, while no differences were observed in the overall cohort, illustrating the potential usefulness of the biomarker in clinical trial design and enrichment in high risk patients. Moreover, urinary proteomics disclosed a pattern of dipeptidyl peptidase-4 inhibition that may be used to assess compliance and monitor the intensity of therapy.

A further review by Marx et al. explores the application of clinical proteomics for identification of molecular pathways specific for diverse, clinically relevant forms of kidney allograft disease such as antibody-mediated rejection, $\mathrm{T}$ cell-mediated rejection, interstitial fibrosis and tubular atrophy, and polyoma virus-associated nephropathy. ${ }^{[14]}$ A well-designed clinical proteomics may be used for noninvasive monitoring and selection of patients that need renal biopsy for adjustment of therapy.

In summary, the current issue of PROTEOMICS-Clinical Applications provides an overview of the state-of-the-art of clinical proteomics in kidney disease, both through a review of available information regarding the impact of preclinical models and urinary proteomics and through novel data on the possibilities offered by urinary proteomics for risk stratification and design of clinical trials. CKD is an example where clinical proteomics effectively delivered, a demonstrator how to evolve from basic science to developing a valuable tool ready for clinical implementation, is expected to have a major impact in patient management.

\section{Acknowledgements}

The author was supported by FIS PI16/02057, ISCIII-RETIC REDinREN RD016/0009 Fondos FEDER, and Comunidad de Madrid B2017/BMD3686 CIFRA2-CM.

\section{Conflict of Interest}

The author declares no conflict of interest.

Received: February 12, 2019

Published online: February 25, 2019

[1] A. Ortiz, M. D. Sanchez-Niño, M. Crespo-Barrio, P. De-SequeraOrtiz, E. Fernández-Giráldez, R. García-Maset, M. Macía-Heras, M. Pérez-Fontán, M. Rodríguez-Portillo, M. Salgueira-Lazo, E. SánchezÁlvarez, R. Santamaría-Olmo, F. Simal-Blanco, M. D. Pino-Pino, Nefrologia 2019, 39, 29.

[2] GBD 2016 Causes of Death Collaborators, Lancet 2017, 390, 1151.

[3] M. V. Perez-Gomez, L. A. Bartsch, E. Castillo-Rodriguez, R. Fernandez-Prado, B. Fernandez-Fernandez, C. Martin-Cleary, C. Gracia-Iguacel, A. Ortiz, Clin. Kidney J. 2019. https://doi. org/10.1093/ckj/sfz007

[4] A. Levin, M. Tonelli, J. Bonventre, J. Coresh, J. A. Donner, A. B. Fogo, C. S. Fox, R. T. Gansevoort, H. J. L. Heerspink, M. Jardine, B. Kasiske, A. Köttgen, M. Kretzler, A. S. Levey, V. A. Luyckx, R. Mehta, O. Moe, G. Obrador, N. Pannu, C. R. Parikh, V. Perkovic, C. Pollock, P. Stenvinkel, K. R. Tuttle, D. C. Wheeler, K. U. Eckardt, Lancet 2017, 390, 1888.

[5] R. L. Mehta, J. Cerdá, E. A. Burdmann, M. Tonelli, G. García-García, V. Jha, P. Susantitaphong, M. Rocco, R. Vanholder, M. S. Sever, D. Cruz, B. Jaber, N. H. Lameire, R. Lombardi, A. Lewington, J. Feehally, F. Finkelstein, N. Levin, N. Pannu, B. Thomas, E. Aronoff-Spencer, G. Remuzzi, Lancet 2015, 385, 2616.

[6] A. Ortiz, A. Covic, D. Fliser, D. Fouque, D. Goldsmith, M. Kanbay, F. Mallamaci, Z. A. Massy, P. Rossignol, R. Vanholder, A. Wiecek, C. Zoccali, Lancet 2014, 383, 1831.

[7] B. Fernandez-Fernandez, M. C. Izquierdo, L. Valiño-Rivas, D. Nastou, A. B. Sanz, A. Ortiz, M. D. Sanchez-Niño, Nephrol. Dial. Transplant. 2018, 33, 1712.

[8] M. D. Sanchez-Niño, A. B. Sanz, A. M. Ramos, B. FernandezFernandez, A. Ortiz, Clin. Kidney J. 2017, 10, 188.

[9] C. Pontillo, H. Mischak, Clin. Kidney J. 2017, 10, 192.

[10] T. Rezk, J. A. Gilbertson, P. P. Mangione, D. Rowczenio, N. Rendell, D. Canetti, H. J. Lachmann, A. D. Wechalekar, P. Bass, P. N. Hawkins, V. Bellotti, G. W. Taylor, J. D. Gillmore, J. Pathol. Clin. Res. 2019. https://doi.org/10.1002/cjp2.126

[11] J. Klein, J. P. Schanstra, Proteome. Clin. Appl. 2018, 13, 1800089.

[12] F. Persson, P. Rossing, Proteome. Clin. Appl. 2019, 13, 1800176.

[13] J. Siwy, T. Klein, M. Rosler, M. von Eynatten, Proteome. Clin. Appl. 2019, 13, 1800144

[14] D. Marx, J. Metzger, J. Olagne, I. Belczacka, S. Faguer, M. Colombat, H. Husi, W. Mullen, W. Gwinner, S. Caillard, Proteome. Clin. Appl. 2019, 13, 1800091.

[15] A. Ortiz, H. Husi, L. Gonzalez-Lafuente, L. Valiño-Rivas, M. Fresno, A. B. Sanz, W. Mullen, A. Albalat, S. Mezzano, T. Vlahou, H. Mischak, M. D. Sanchez-Niño, J. Am. Soc. Nephrol. 2017, 28, 823.

[16] N. Tofte, M. Lindhardt, K. Adamova, J. Beige, J. W. J. Beulens, A. L. Birkenfeld, G. Currie, C. Delles, I. Dimos, L. Francová, M. FrimodtMøller, P. Girman, R. Göke, T. Havrdova, A. Kooy, H. Mischak, G. Navis, G. Nijpels, M. Noutsou, A. Ortiz, A. Parvanova, F. Persson, P. L. Ruggenenti, F. Rutters, I. Rychlík, G. Spasovski, M. Speeckaert, M. Trillini, H. von der Leyen, Rossing P, Diabet. Med. 2018, 35, 1375.

[17] M. E. Rodríguez-Ortiz, C. Pontillo, M. Rodríguez, P. Zürbig, H. Mischak, A. Ortiz, Sci. Rep. 2018, 8, 15940. 